\title{
Evaluating the extent of agreement between the EARP (Early Arthritis for Psoriatic Patients) and PEST (Psoriasis Epidemiology Screening Tool)
} questionnaires in screening for psoriatic arthropathy in patients with psoriasis in a tertiary-care dermatology outpatient department

\author{
Suyash Singh ${ }^{1}$, Aradhana Sood ${ }^{2}$, Preema Sinha ${ }^{3}$, Rajneesh Joshi ${ }^{4}$, Seema Patrikar, \\ Pankaj Das ${ }^{6}$
}

${ }^{1}$ Undergraduate student, Armed Forces Medical College, Pune, Maharashtra, India, ${ }^{2}$ Professor and HOD Department of Dermatology, Armed Forces Medical College, Pune, Maharashtra, India, ${ }^{3}$ Assoc Professor, Department of Dermatology, Armed Forces Medical College, Pune, Maharashtra, India, ${ }^{4}$ Professor, Department of Community Medicine, Armed Forces Medical College, Pune, Maharashtra, India, ${ }^{5}$ Lecturer in Statistics, Department of Community Medicine, Armed Forces Medical College, Pune, Maharashtra, India, ${ }^{6}$ Senior resident, Department of Dermatology, Armed Forces Medical College, Pune, Maharashtra, India

Corresponding author: Major Pankaj Das, E-mail: pankaj3609@gmail.com

\begin{abstract}
Background: The prevalence of psoriatic arthropathy (PsA) among psoriatic patients ranges from $5 \%$ to $42 \%$, with some cases of rapidly progressive disabling arthropathy. If detected early, PsA can be considerably improved by timely therapeutic intervention. Various screening tools have been developed to screen psoriatic patients for arthritis, but there is a paucity of literature on the agreement between the two. Aim: Evaluating the extent of agreement between the EARP (Early Arthritis for Psoriatic Patients) and PEST (Psoriasis Epidemiology Screening Tool) questionnaires in screening psoriatic patients for psoriatic arthropathy in a tertiary-care dermatology outpatient department (OPD). Materials and Methods: 100 prospective psoriatic patients with no prior diagnosis of PsA reporting to our dermatology OPD were administered EARP and PEST questionnaires. The extent of agreement between the two questionnaires was calculated by Cohen's kappa coefficient. Those positive for PsA by one or both of the questionnaires were evaluated using the CASPAR criteria. Results: 43 patients were positive for PsA by EARP, whereas 13 patients were positive by PEST; and all of these 13 patients were EARP positive as well. All the patients who were either EARP or PEST positive continued to meet the CASPAR criteria, showing a positive predictive value of $100 \%$ for both questionnaires. The extent of agreement between EARP and PEST was found to be low (0.312). Conclusion: EARP is a better screening tool for PsA than PEST, as the latter failed to screen positively a significant number of psoriatic patients for psoriatic arthropathy. The extent of agreement between the two questionnaires can, thus, be considered poor.
\end{abstract}

Key words: Psoriasis; Psoriatic arthropathy; EARP; PEST; CASPAR

\section{INTRODUCTION}

Psoriasis is a common chronic relapsing inflammatory disease with dermatological as well as systemic manifestations. The estimated worldwide prevalence of psoriasis ranges from $0.51 \%$ to $11.43 \%$, while the prevalence in India varies from $0.44 \%$ to $2.8 \%[1,2]$. Psoriasis is currently considered a multisystem disorder

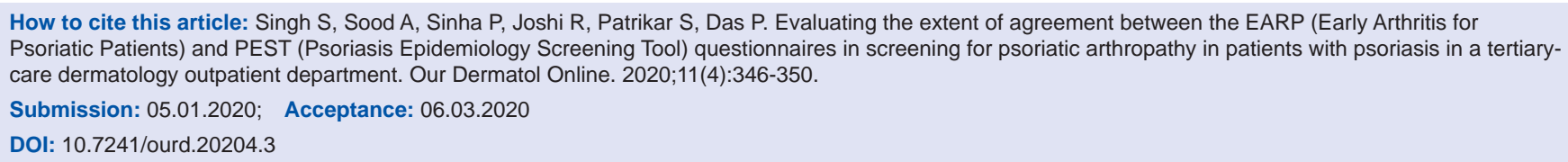


with chronic inflammation linked with psoriatic arthropathy, obesity, hypertension, dyslipidemia, and insulin resistance [3]. Psoriatic arthropathy can be acutely disabling, leading to a poor quality of life (QoL).

There is now enough evidence to believe that early diagnosis of PsA may prevent disabling arthropathy [4]. Since, in most patients with psoriasis, skin lesions precede those of joint involvement, dermatologists are at an advantageous position to screen patients for PsA [5]. In the past few years, numerous screening tools for PsA detection have been devised: Toronto Psoriatic Arthritis Screening Questionnaire-II (TOPAS-II), Psoriatic Arthritis Screening and Evaluation (PASE), Psoriasis Epidemiology Screening Test (PEST), and Early Arthritis for Psoriasis Patients (EARP) [6]. Among the above four, EARP and PEST are simple, self-administered, and easy-to-use questionnaires that can be used in busy dermatology OPDs to screen patients for PsA (Tables 1 and 2). We tested the extent of agreement between these two questionnaires in a tertiary-care dermatology center in western India and their positive predictive values.

\section{MATERIALS AND METHODS}

\section{Study Design}

The following cross-sectional study was conducted in one of the tertiary care dermatology OPDs in western

Table 1: EARP questionnaire (scores of 3 or above are positive).

\begin{tabular}{lcc}
\hline Question & Yes & No \\
\hline $\begin{array}{l}\text { Do your joints hurt? } \\
\text { Have you taken anti-inflammatory more than twice a week for }\end{array}$ & 1 & 0 \\
joint pain in the last 03 months? & & \\
Do you wake up at night because of low back pain? & 1 & 0 \\
Do you feel stiffness in your hands for more than 30 minutes & 1 & 0 \\
in the morning? & & \\
Do your wrists and fingers hurt? & 1 & 0 \\
Do your wrists and fingers swell? & 1 & 0 \\
Does one finger hurt and swell for more than 03 days? & 1 & 0 \\
Does your Achilles tendon swell? & 1 & 0 \\
Do your feet or ankles hurt? & 1 & 0 \\
Do your elbow or hips hurt? & 1 & 0 \\
\hline
\end{tabular}

Table 2: PEST questionnaire (scores of 3 or above are positive).

\begin{tabular}{lcc}
\hline Question & Yes & No \\
\hline Have you ever had a swollen joint (or joints)? & 1 & 0 \\
Has a doctor ever told you that you have arthritis? & 1 & 0 \\
Do your finger nails or toe nails have holes or pits? & 1 & 0 \\
Have you had pain in your heel? & 1 & 0 \\
Have you had a finger or toe that was completely swollen and & 1 & 0 \\
painful for no apparent reason? & & \\
\hline
\end{tabular}

(c) Our Dermatol Online 4.2020
India from September 2018 to March 2019. Ethical approval was obtained from an institutional ethical committee. 100 patients were selected from the dermatology OPD over a period of 6 months according to the inclusion and exclusion criteria of the study.

\section{Inclusion Criteria}

All diagnosed cases of psoriasis visiting the dermatology OPD were included in the study.

\section{Exclusion Criteria}

All previously diagnosed cases of psoriatic arthropathy, rheumatoid arthritis, gout, and other rheumatic diseases were excluded from the study.

\section{Methodology}

The participants were provided with an informed assent sheet highlighting the purpose, methodology, risks, benefits, and confidentiality of the study as well as the right to refuse participation. All patients willing to participate and having met the inclusion and exclusion criteria and filled a written informed consent were enrolled in the study. The enrolled patients were asked to fill EARP and PEST questionnaires along with a predesigned basic disease data sheet. Parents were asked to consent and fill the questionnaires on behalf of patients younger than 18. Patients scoring positive in either the EARP or PEST questionnaire or both underwent radiography, and were tested for RA factor in order to meet the CASPAR criterion of psoriatic arthropathy (Table 3).

\section{Statistical Analysis}

Data analysis was performed using the software SPSS Statistics for Windows, version 20.0. Armonk, NY: IBM Corp. The extent of agreement between the results of the two questionnaires was calculated by Cohen's kappa coefficient.

\section{Ethics Statement}

Ethical approval was obtained from an institutional ethical committee.

\section{RESULTS}

Of the 100 patients enrolled in our study, 76 were males and 24 were females. The mean age was 47 years, 


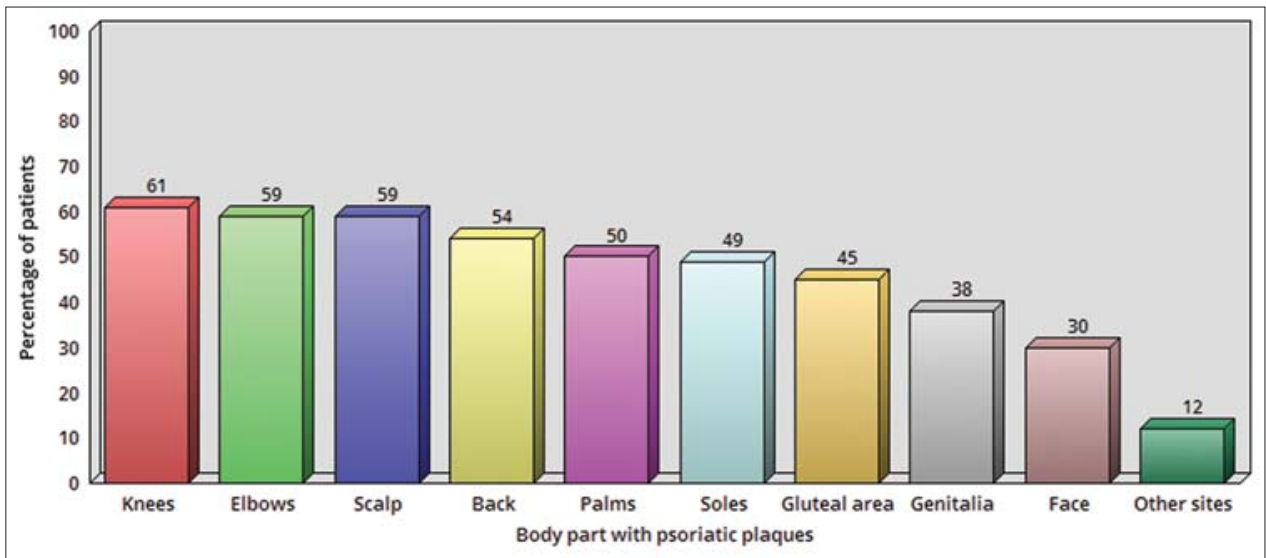

Figure 1: Distribution of patients according to the part of the body affected by psoriatic lesions.

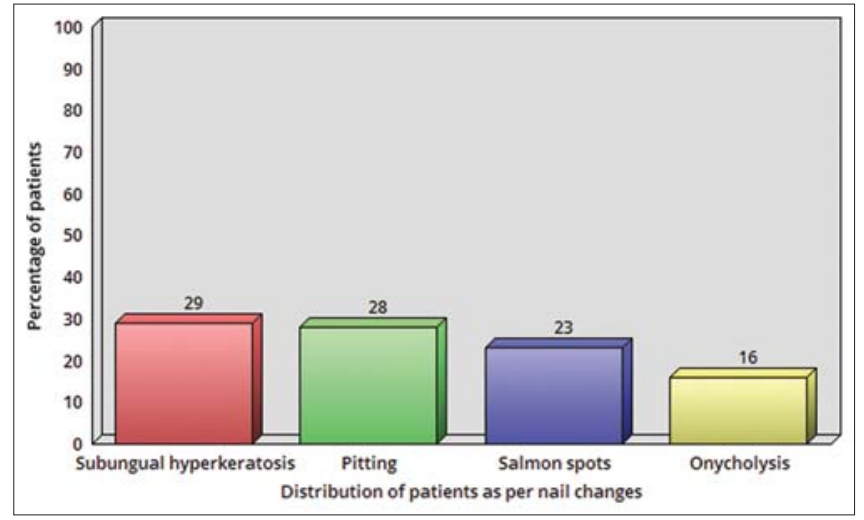

Figure 2: Distribution of patients according to nail changes.

ranging between 9 and 79 years. The patients had been suffering from psoriasis for an average duration of 7 years, with a minimum and maximum duration of 40 days and 31 years, respectively.

The distribution of patients according to the sites affected by psoriatic lesions is depicted in Fig. 1. $40 \%$ of the patients were found to have changes in nail structure and architecture, which are associated with psoriasis. The distribution of patients according to the type of nail involvement is shown in Fig. $2.45 \%$ of the patients reported to suffer from some type of joint pain. The distribution of patients according to the different joints involved is shown in Fig. 3. Joint pain had a progressive course in $22 \%$ of the patients. $40 \%$ of the patients had stiffness of the joints at some point of time during the day. Among them, 34\% complained of joint stiffness in early morning hours; $12 \%$ while asleep; and $8 \%$ and $2 \%$ complained of most joint stiffness during afternoon and evening hours, respectively. In an analysis of the EARP and PEST questionnaires, 43 out of the 100 enrolled patients scored positive for $\operatorname{EARP}(\geq 3)$, while 13 patients scored positive for $\operatorname{PEST}(\geq 3)$; all of these 13 patients were EARP positive as well. All those
Table 3: The Classification Criteria for Psoriatic Arthritis (CASPAR) for diagnosis of psoriatic arthropathy.

\begin{tabular}{|c|c|}
\hline Criteria & Point value \\
\hline Current psoriasis & 2 \\
\hline Personal history of psoriasis & 1 \\
\hline Family history of psoriasis & 1 \\
\hline $\begin{array}{l}\text { Typical psoriatic nail dystrophy (onycholysis, pitting, } \\
\text { hyperkeratosis) }\end{array}$ & 1 \\
\hline Negative rheumatoid factor & 1 \\
\hline $\begin{array}{l}\text { Current dactylitis or history of dactylitis (recorded by } \\
\text { rheumatologist) }\end{array}$ & 1 \\
\hline $\begin{array}{l}\text { Hand or foot plain radiography: evidence of juxta-articular } \\
\text { new bone formation, appearing as ill-defined ossification } \\
\text { near joint margins (excluding osteophytes) }\end{array}$ & 1 \\
\hline
\end{tabular}

who were either EARP or PEST positive continued to meet the CASPAR criteria, which is the gold standard for diagnosis of PsA, showing a positive predictive value of $100 \%$. The extent of agreement between EARP and PEST, as calculated by Cohen's kappa coefficient, was 0.312. In our study, EARP was superior to PEST, as PEST failed to screen positively a significant number of psoriatic patients for psoriatic arthropathy.

\section{DISCUSSION}

Psoriasis is one of the most common diseases examined in dermatology OPDs. Chronic systemic inflammation associated with psoriasis results in its association with metabolic syndrome, in complications, and in psoriatic arthropathy. The prevalence of inflammatory arthritis in the general population is 2-3\%, whereas the prevalence in psoriasis is 6-42\% [7]. The prevalence of psoriasis in the world population is estimated at 2-3\%. Depending on genetic susceptibility and the geographic region, the prevalence of PsA ranges from 5\% to $42 \%$ [8]. In a multiethnic retrospective study conducted at a rheumatology and dermatology referral center in Singapore, PsA was found to be significantly more 


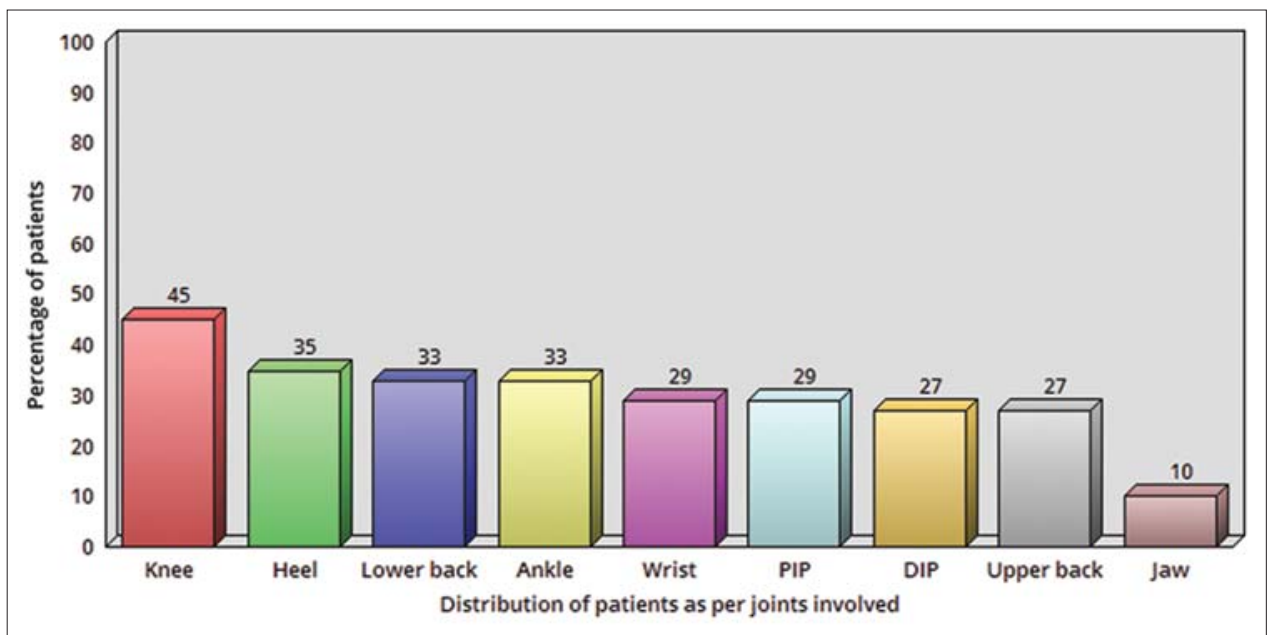

Figure 3: Distribution of patients according to joint involvement.

common among the Indian population as compared to people of other ethnicities ( $<<0.000001)$ [9].

The spectrum of disease activity of PsA ranges from a benign course with mild symptoms to a rapidly destructive course resulting in permanent disability and a poor QoL [10]. In 75\% of the patients, psoriatic skin lesions preceded joint symptoms, proving the appropriateness of diagnosing PsA in dermatology OPDs [8]. Presently, the gold standard for diagnosing PsA is the CASPAR criteria, which have a sensitivity of 91.4\% and a specificity of $98.7 \%$ [11]. The CASPAR criteria were designed to be used by physicians and investigators, and requires serological testing of RA factor and bone radiography to have all its criteria met. A busy dermatology OPD may preclude dermatologists from asking each and every psoriatic patient about joint symptoms. Moreover, patients may not associate joint symptoms with psoriasis and may be less likely to report joint pain to a dermatologist, instead receiving drugs and alternative medicine for undiagnosed PsA from other sources, leading to a delayed diagnosis and the negative consequences of PsA. EARP and PEST are simple, objective, and self-administered screening tools that can help with screening psoriatic patients for PsA in dermatology OPDs. However, there have only been a few studies comparing the whole variety of these tools. We tested these screening tools in our dermatology OPD for their extent of agreement and positive predictive values. In our study, $76 \%$ of the subjects were males, as compared to 85\% in Prasad et al. [12], 63\% in Kumar et al. [13], and 51\% in Karreman et al. [5]. The mean age of the patients in our study was 47 years, as compared to 33.1 years in Kumar et al. [13] and 55.7 years in Karreman et al. [5]. The average duration of psoriasis in our study was found to be less (7 years) than in other studies: 9.88 years in Kumar et al. [13] and 20.7 years in Karreman et al. [5]. Joint involvement in our study was classified according to Moll and Wright's criteria: polyarthritis (32\%), spondyloarthropathy (31\%), asymmetric oligoarthritis (28\%), predominant distal interphalangeal (DIP) joint involvement (24\%), and arthritis mutilans (1\%). Kumar et al. reported that the most common pattern of PsA is polyarthritis (58\%), followed by spondyloarthropathy (49\%), asymmetric oligoarthritis $(21 \%)$, predominant DIP arthritis (3\%), and arthritis mutilans (1\%) [13]. Nail involvement is an often-overlooked manifestation of psoriasis and affects approximately 10-78\% of psoriatic patients, with 5-10\% suffering from isolated nail psoriasis [14]. Our study showed that $40 \%$ of the psoriasis patients had nail structure and architecture changes: subungual hyperkeratosis (29\%), pitting (28\%), salmon spots $(23 \%)$, and onycholysis (16\%). Prasad et al. [12] reported that the most common nail change is pitting, followed by subungual hyperkeratosis; while Kumar et al. [13] found pitting to be the most common, followed by onycholysis and subungual hyperkeratosis. In our study, $41.8 \%$ of the patients with psoriatic arthropathy had nail changes. The reported frequency of nail involvement in patients with PsA is $87 \%$ according to Kumar et al. [13] and 63\% according to Scher et al. [15]. Our study showed that EARP was superior to PEST, as PEST failed to screen positively a significant number of psoriatic patients for psoriatic arthropathy.

Our study was limited to the small sample of 100 patients. Moreover, we were not able determine the true and false negatives of the two questionnaires, since we did not further evaluate the subjects who were screened 
negative by the questionnaires, in order to reduce the risk of unnecessary X-ray exposure. Due to a lack of data on the true and false negatives, it was impossible to calculate the sensitivity and specificity of the two questionnaires in our study.

\section{CONCLUSION}

Our study yielded findings commensurate with previous studies, showing that EARP is a more sensitive screening tool for PsA than PEST. However, the poor agreement between the two questionnaires underlines the need for the development of more effective PsA screening tools.

\section{ACKNOWLEDGEMENTS}

- Major Arunjeet Bhan, Assistant Director of Health, I Corps Headquarters, Indian Army

- Anirban Nandy, Data Scientist, Pune, India

- Statement of Human and Animal Rights

All the procedures followed were in accordance with the ethical standards of the responsible committee on human experimentation (institutional and national) and with the 2008 revision of the Declaration of Helsinki of 1975.

\section{Statement of Informed Consent}

Informed consent for participation in this study was obtained from all patients.

\section{REFERENCES}

1. Michalek IM, Loring B, John SM. A systematic review of worldwide epidemiology of psoriasis. J Eur Acad Dermatol Venereol.
2017;31:205-12.

2. Dogra S, Yadav S. Psoriasis in India: Prevalence and pattern. Indian J Dermatol Venereol Leprol. 2010;76:595-603.

3. Gelfand JM, Yeung H. Metabolic syndrome in patients with psoriatic disease. J Rheum Suppl. 2012;89:24-8.

4. Haroon M, Gallagher P, FitzGerald O. Diagnostic delay of more than 6 months contributes to poor radiographic and functional outcome in psoriatic arthritis. Ann Rheum Dis. 2015;74:1045-50.

5. Karreman MC, Weel AE, Van der ven M, Vis M, Tchetverikov I, Nijsten TE et al. Performance of screening tools for psoriatic arthritis: a cross-sectional study in primary care. Rheum. 2016;56:597-602.

6. Mishra S, Kancharla H, Dogra S, Sharma A. Comparison of four validated psoriatic arthritis screening tools in diagnosing psoriatic arthritis in patients with psoriasis. Br J Dermatol. 2017;176:765-70.

7. Gudu T, Gossec L. Quality of life in psoriatic arthritis. Expert Rev Clin Immunol. 2018;14:405-17.

8. Goldenstein-Schainberg C, Favarato MH, Ranza R. Current and relevant concepts in psoriatic arthritis. Rev Bras Rheum. 2012;52:98-106.

9. Solmaz D, Eder L, Aydin SZ. Update on the epidemiology, risk factors, and disease outcomes of psoriatic arthritis. Best Pract Res Clin Rheumatol. 2018;32:295-311.

10. Cuchacovich R, Perez-Alamino R, Garcia-Valladares I, Espinoza LR. Steps in the management of psoriatic arthritis: a guide for clinicians. Therapeutic Adv Chronic Dis. 2012;3:259-69.

11. Zhang A, Kurtzman DJB, Perez-Chada LM, Merola JF. Psoriatic arthritis and the dermatologist: an approach to screening and clinical evaluation. Clin Dermatol. 2018;36:551-60.

12. Prasad PVS, Babu B, Kaviarasan PK, Senthilnathan A. A Clinical Study of Psoriatic arthropathy. Indian J Dermatol Venereol Leprol. 2007;73:166-70.

13. Kumar R, Sharma A, Dogra S. Prevalence and clinical patterns of psoriatic arthritis in Indian patients with psoriasis. Indian J Dermatol Venereol Leprol. 2014;80:15-23.

14. Dogra A, Arora AK. Nail psoriasis: The journey so far. Indian J Dermatol. 2014;59:319-33.

15. Scher JU, Ogdie A, Merola JF, Ritchlin C. Preventing psoriatic arthritis: focusing on patients with psoriasis at increased risk of transition. Nat Rev Rheumatol. 2019;15:153-66.

Copyright by Suyash Singh, et al. This is an open access article distributed under the terms of the Creative Commons Attribution License, which permits unrestricted use, distribution, and reproduction in any medium, provided the original author and source are credited.

Source of Support: Nil, Conflict of Interest: None declared. 